\title{
Enabling Dominance Resistance in Visualisable Distance-Based Many-Objective Problems
}

\author{
Jonathan E. Fieldsend \\ Computer Science \\ University of Exeter \\ Exeter, UK, EX4 4QF \\ J.E.Fieldsend@exeter.ac.uk
}

\begin{abstract}
The results when optimising most multi- and many-objective problems are difficult to visualise, often requiring sophisticated approaches for compressing information into planar or 3D representations, which can be difficult to decipher. Given this, distance-based test problems are attractive: they can be constructed such that the designs naturally lie on the plane, and the Pareto set elements easy to identify. As such, distance-based problems have gained in popularity as a way to visualise the distribution of designs maintained by different optimisers. Some taxing problem aspects (many-to-one mappings and multi-modality) have been embedded into planar distance-based test problems, although the full range of problem characteristics which exist in other test problem frameworks (deceptive fronts, degeneracy, etc.) have not. Here we present an augmentation to the distance-based test problem formulation which induces dominance resistance regions, which are otherwise missing from these test problems. We illustrate the performance of two popular optimisers on test problems generated from this framework, and highlight particular problems with evolutionary search that can manifest due to the problem characteristics.
\end{abstract}

\section{Keywords}

Multi-objective test problems; dominance resistance points; niching; evolutionary optimisation.

\section{INTRODUCTION}

Visualising a Pareto front approximation delivered by an evolutionary algorithm (EA), along with the associated Pareto set approximation (and the relationship between them) is often difficult. This is because often one or both sets inhabit a space whose dimension is greater than three (the space which humans naturally visualise and interact with). A number of approaches to enable the visualisation of designs in these domains have been utilised over the years. This often includes 'traditional' visualisations like parallel coordinate plots (an approach which dates back to at least

Permission to make digital or hard copies of all or part of this work for personal or classroom use is granted without fee provided that copies are not made or distributed for profit or commercial advantage and that copies bear this notice and the full citation on the first page. Copyrights for components of this work owned by others than the author(s) must be honored. Abstracting with credit is permitted. To copy otherwise, or republish, to post on servers or to redistribute to lists, requires prior specific permission and/or a fee. Request permissions from permissions@ acm.org.

GECCO'16 Companion, July 20 - 24, 2016, Denver, CO, USA

(C) 2016 Copyright held by the owner/author(s). Publication rights licensed to ACM. ISBN 978-1-4503-4323-7/16/07 . \$ \$15.00

DOI: http://dx.doi.org/10.1145/2908961.2935616 the late $1800 \mathrm{~s}$, see plate 151 of [5]) and heatmaps [18, 16]. More recently specialised approaches specifically developed for multi-objective data have started to be used. For instance scatterplot approaches which compress information but attempt to preserve domination relationships explicitly $[12,2]$, or via a distance [20].

Although popular, it is quite difficult to ascertain from visualisations such as parallel coordinate plots and heatmaps the distribution of solutions. This is exacerbated as the number of dimensions (in either space) increases, and also as the number of solutions considered increases. Alternatively, specialised scatterplot visualisation approaches tend to lose information due to their data compression from a higher number of dimensions into the two or three dimension used to visualise the data. Distance-based multi- and many-objective problems, first popularised in [10, 11] for visualisation, sidestep these issues. They formulate problems which can have arbitrarily many objectives, but whose design space natively lives in two-dimensions - where the Pareto set is easy to identify visually. These problems have been used in a number of empirical studies (e.g. [17, 8, 14]) in order to visualise the distribution of designs maintained by multi- and many-objective optimisers during their search - providing qualitative information. In the distance-based formulation (also sometimes referred to as a Pareto-box formulation), a putative solution is a point in the plane, and its performance on each objective is calculated as its distance to a point in that space. (In later extensions, the minimum distance to an element of a set is also used.) A problem instance is therefore parameterised by the set of points, $\left\{\mathbf{v}_{i}\right\}$, used in the objective calculations for proposed solutions, along with a distance metric, $\operatorname{dist}(\cdot, \cdot)$ (usually Euclidean).

The original framework has been extended in the last few years to facilitate such properties as many-to-one mapping, multi-modality and arbitrarily many design variables - whilst still retaining the key property of being easy to visualise in the plane $[6,7,9,13,15]$. Recent work has also investigated the use of non-Euclidean distance metrics within this framework, and the identification of the Pareto optimal set in these situations [21].

\section{PARETO OPTIMALITY}

Without loss of generality, when optimising a multiobjective problem we seek to simultaneously minimise $D$ objectives: $f_{d}(\mathbf{x}), d=1, \ldots, D$ where each objective depends upon a vector $\mathbf{x}=\left(x_{1}, \ldots, x_{K}\right)$ of $K$ parameters or decision variables. These parameters may also be subject to equality and inequality constraints, which together define $\mathcal{X} \in \mathbb{R}^{K}$, 
the feasible search space. Related to this is $\mathcal{Y}$, the objective space image of $\mathcal{X}$ (sometimes referred to as the feasible objective space). When faced with only a single objective an optimal solution $\mathbf{x}^{*}$ is one which minimises the objective, subject to $\mathbf{x}^{*} \in \mathcal{X}$. However, when there is more than one objective to be minimised, solutions may exist for which performance on one objective cannot be improved without reducing performance on at least one other. Such solutions are said to be Pareto optimal. The set of all Pareto optimal solutions is said to form the Pareto set, $\mathcal{P}$, whose image in objective space is known as the Pareto front, $\mathcal{F}$.

A decision vector $\mathbf{x}$ is said to dominate another $\mathbf{x}^{\prime}$ iff

$$
f_{d}(\mathbf{x}) \leq f_{d}\left(\mathbf{x}^{\prime}\right) \quad \forall d=1, \ldots, D \quad \text { and } \quad \mathbf{f}(\mathbf{x}) \neq \mathbf{f}\left(\mathbf{x}^{\prime}\right)
$$

This is often denoted as $\mathbf{x} \prec \mathbf{x}^{\prime}$. Multi- and many-objective evolutionary algorithms ('many' objectives is generally referred to in the literature as four or more) typically maintain a set of mutually non-dominating solutions $A$. This set is usually referred to as an archive, and forms their estimated Pareto set. This may be active (providing input into the optimisation process) or a passive record of the best solutions ever encountered during the optimisation [19]. It is useful when examining the properties of an optimiser to look at the distribution of designs in the image of $A$, as one characteristic often preferred in an optimiser is for the designs to be evenly distributed on the approximation to $\mathcal{F}$ - providing the decision maker a 'good' range of designs to choose from, given $|A|$.

During the development of new optimisers, it is often useful to apply them to test problems whose properties are similar in some way to the real-world problem the optimiser is being developed for. Test problems can also help us examine how an algorithm tends to behave, and visualising this can be very useful. This has led to the development of distancebased test problems to examine the population dynamics and diversity preservation properties of optimisers.

\section{DISTANCE-BASED PROBLEMS}

In the standard formulation, the $d$ th distance-based objective (criterion) is a function of a putative design $\mathbf{x}$, a set of $w$ vertices $V_{d}=\left\{\mathbf{v}_{i}\right\}_{i=1}^{w}$ defined for that objective, and a distance metric $\operatorname{dist}()$, such that

$$
f_{d}(\mathbf{x})=\min _{\mathbf{v} \in V_{d}}(\operatorname{dist}(\mathbf{x}, \mathbf{v}))
$$

In [10] $\left|V_{d}\right|=1$, however later extensions to the distancebased framework (e.g. [8]) incorporate a set of comparison vertices per objective as a way to induce disjoint Pareto sets (a multi-modal landscape).

Fig. 1 provides a simple illustration of a $D=2$ distancebased test problem. In the left panel $\mathcal{X}$ is shown: $K=2$, the inequality constraints bound the feasible search space to the unit square, $V_{1}=\{(0.45,0.5)\}$ and $V_{2}=\{(0.55,0.5)\}$. Using the Euclidean distance, $\mathcal{P}$ is the line segment joining $(0.45,0.5)$ and $(0.55,0.5)$. In the right panel the corresponding $\mathcal{Y}$ is shown (via evaluating 10,000 samples uniformly drawn from $\mathcal{X})$.

It can be seen in Fig. 1 that the $\mathcal{Y}$ induced by the standard formulation closes in on the extremes, such that a solution which minimises one objective, is always a member of the Pareto set. This is the case irrespective of $D$. Furthermore, due to recommended use of $V_{d}$ in the literature - to form separate regions of Pareto optimal solutions in $\mathcal{X}$, which are

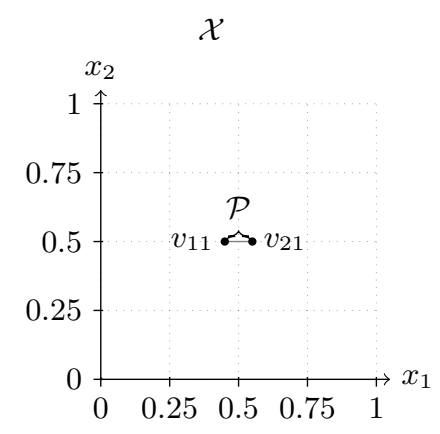

$\mathcal{Y}$

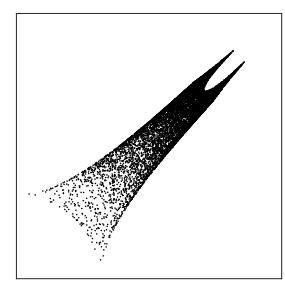

Figure 1: Test problem illustration. Left: Objectives derived from distance to points, visualisation of $\mathcal{X}$ with $\mathcal{P}$ highlighted. Right: corresponding $\mathcal{Y}$.
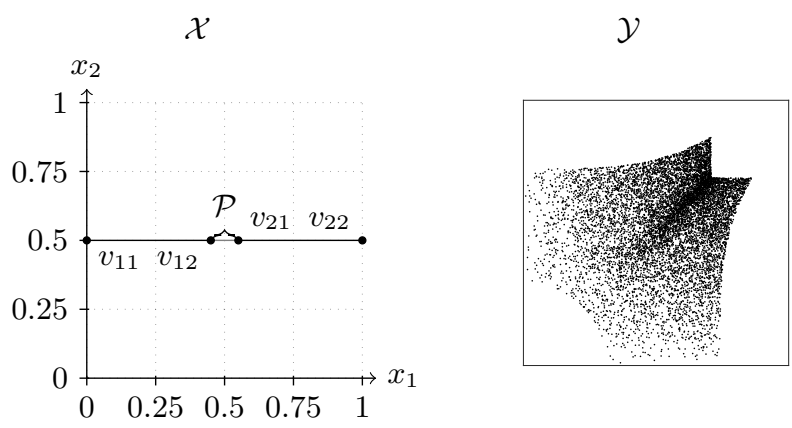

Figure 2: Test problem illustration. Left: Objectives derived from distance to line segments, visualisation of $\mathcal{X}$ with $\mathcal{P}$ highlighted. Right: corresponding $\mathcal{Y} . \mathcal{P}$ is the same as in Fig. 1 , but $\mathcal{Y}$ no longer contracts down at the edges of the Pareto front.

not too close [6] - this is also the case for the commonly used disjoint Pareto set mappings.

In many real world problems designs which minimise one quality criterion (or are close to the minimum on a criterion), are often not Pareto optimal, and lead in turn to the dominance resistance points problem for multi-objective optimisers [4]. This is where optimisers can be drawn to search around points (designs) that are very far from $\mathcal{F}$ (and likely also $\mathcal{P}$ ) due to their good performance on one (or a handful of criteria), albeit very poor performance on others. This issue does not arise in the current construction of visual distance-based problems, as any solution that minimises one criteria (or is close to it), is in (or close to) $\mathcal{F}$ and $\mathcal{P}$. It is the provision of dominance resistance solutions in visual distance-based multi-objective test problems which we now consider.

\section{DOMINANCE RESISTANCE}

One mechanism to enable dominance resistance points, is to replace distances from vertices in (2), to distances from lines/regions. An illustration of the effect of this is shown in Fig. 2. This has the same $\mathcal{P}$ as Fig. 1, however the vertices for the two criteria at $(0.45,0.5)$ and $(0.55,0.5)$ have been replaced by line segments (for criterion 1 , from $(0.0,0.5)$ to $(0.45,0.5)$, and for criterion 2 from $(0.55,0.5)$ to $(1.0,0.5))$. 

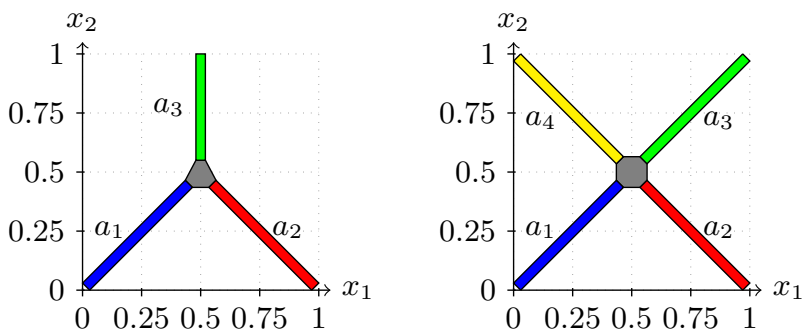

Figure 3: Test problems using distance to regions. Minima regions for each criterion in colour and $\mathcal{P}$ in grey.
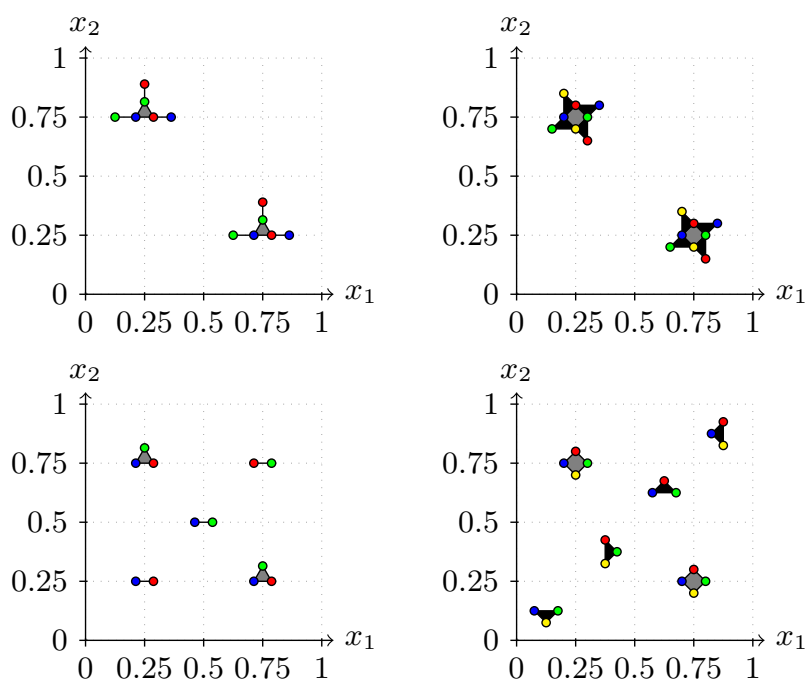

Figure 4: Example test problems. Vertices highlighted with filled circles (colour denoting criterion vertex minimises). Dominance resistance areas in black and $\mathcal{P}$ in grey. Left column: Three objectives. Right column: Four objectives. Top row: Dominance resistance areas contiguous with $\mathcal{P}$. Bottom row: Dominance resistance areas and $\mathcal{P}$ in separate regions.

The effect on $\mathcal{Y}$ can be seen in the right panel of Fig. 2, where the mapping no longer pinches at the extremes of $\mathcal{F}$. It is worth noting here that a coloured region in Fig. 2 contains designs which are dominated by other designs in the same region, unlike the grey region denoting $\mathcal{P}$, which is induced by the coloured regions, but which contains only mutually non-dominating solutions.

Although this modification has the effect we desire in the $D=2$ situation, it becomes geometrically limited as $D$ increases. Fig. 3 illustrates examples using this approach for $D=3$ and $D=4$ problems. The areas with minimum criteria values are denoted $a_{d}$ and coloured according to minimised criteria. Any solution in $a_{d}$ has the minimum value of 0.0 for the $d$ th criterion, and the minimum distance to any of the lines making up its sides is used to compute the quality on the $d$ th criterion of a solution in $\mathcal{X}$ which lies outside this area. The effect of this is to have dominance

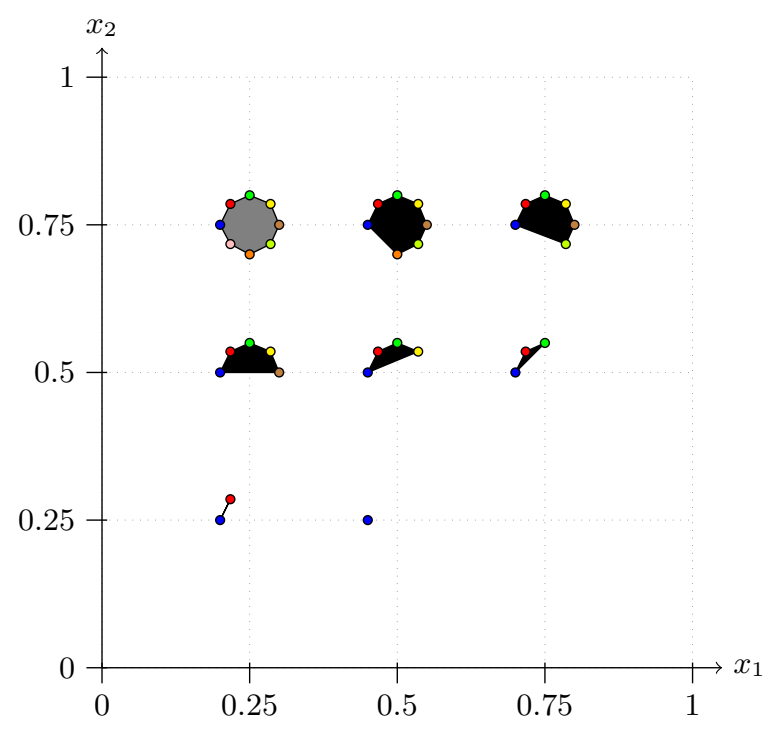

Figure 5: $D=8$ problem. Vertices highlighted with filled circles (colour denoting criterion vertex minimises).
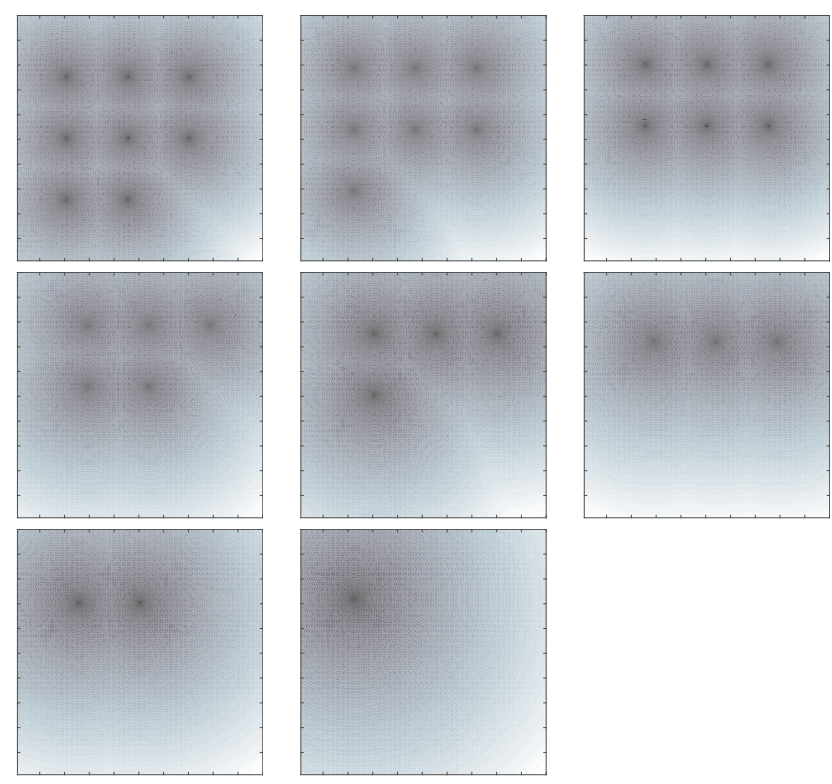

Figure 6: Cost landscape in $\mathcal{X}$ induced by each objective from the problem shown in Fig. 5. (Darker regions denote lower cost.)

resistant solutions which have the minimum (or near the minima) on one criteria. However it does not provide dominance resistant points with multiple good quality values. This property could be provided via designing parts of these resistance areas to be close to each in regions away from $\mathcal{P}$, but such construction has to be careful (and can result in a large proportion of $\mathcal{X}$ being a dominant resistant region). A more elegant approach can instead be found by revisit- 
Table 1: Distance-based test problems with dominance resistance areas used in empirical section.

\begin{tabular}{|c|c|}
\hline$\pi$ & Probles \\
\hline 1 & $\begin{array}{c}V_{1}=\{(0.2125,0.75),(0.7125,0.25),(0.3625,0.75) \\
(0.8625,0.25)\} \\
V_{2}=\{(0.2875,0.75),(0.7875,0.25),(0.25,0.88995) \\
(0.75,0.38995)\} \\
V_{3}=\{(0.250 .81495),(0.75,0.31495),(0.125,0.75) \\
(0.625,0.25)\}\end{array}$ \\
\hline 2 & $\begin{array}{c}V_{1}=\{(0.2125,0.75),(0.7125,0.25),(0.2125,0.25) \\
(0.4625,0.5)\} \\
V_{2}=\{(0.2875,0.75),(0.7875,0.25),(0.2875,0.25) \\
(0.7125,0.75)\} \\
V_{3}=\{(0.25,0.81495),(0.75,0.31495),(0.5375,0.5) \\
(0.7875,0.75)\}\end{array}$ \\
\hline 3 & $\begin{array}{l}V_{1}=\{(0.2,0.75),(0.7,0.25),(0.35,0.8),(0.85,0.3)\} \\
V_{2}=\{(0.25,0.8),(0.75,0.3),(0.3,0.65),(0.8,0.15)\} \\
V_{3}=\{(0.3,0.75),(0.8,0.25),(0.15,0.7),(0.65,0.2)\} \\
V_{4}=\{(0.25,0.7),(0.75,0.2),(0.2,0.85),(0.7,0.35)\}\end{array}$ \\
\hline 4 & $\begin{aligned} & V_{1}=\{(0.2,0.75),(0.7,0.25),(0.075,0.125), \\
&(0.575,0.625),(0.825,0.875)\} \\
& V_{2}=\{(0.25,0.8),(0.75,0.3),(0.375,0.425), \\
&(0.625,0.675),(0.875,0.925)\} \\
& V_{3}=\{(0.3,0.75),(0.8,0.25),(0.175,0.125), \\
&(0.425,0.375),(0.675,0.625)\} \\
& V_{4}=\{(0.25,0.7),(0.75,0.2),(0.125,0.075), \\
&(0.375,0.325),(0.875,0.825)\}\end{aligned}$ \\
\hline 5 & $\begin{array}{c}V_{1}=\{(0.2,0.75),(0.45,0.75),(0.7,0.75),(0.2,0.5), \\
(0.45,0.5),(0.7,0.5),(0.2,0.25),(0.45,0.25)\} \\
V_{2}=\{(0.2174,0.78535),(0.4674,0.78535), \\
(0.7174,0.78535),(0.2174,0.53535),(0.4674,0.53535), \\
(0.7174,0.53535),(0.2174,0.28535)\} \\
V_{3}=\{(0.25,0.8),(0.5,0.8),(0.75,0.8),(0.25,0.55), \\
(0.5,0.55),(0.75,0.55)\} \\
V_{4}=\{(0.28535,0.78535),(0.53535,0.78535), \\
(0.78535,0.78535),(0.28535,0.53535), \\
(0.53535,0.53535)\} \\
V_{5}=\{(0.3,0.75),(0.55,0.75),(0.8,0.75),(0.3,0.5)\} \\
V_{6}=\{(0.28535,0.7174),(0.53535,0.7174), \\
(0.78535,0.7174)\} \\
V_{7}=\{(0.25,0.7),(0.5,0.7)\} \\
V_{8}=\{(0.2174,0.7174)\}\end{array}$ \\
\hline
\end{tabular}

ing the methodology for generating multiple Pareto optimal regions in $\mathcal{X}$.

Rather than using vertices simply to bound the Pareto optimal region in $\mathcal{X}, D-m$ vertices (for $D-m$ objectives) may be used, where $0<m<D$. These vertices bound dominance resistance areas, $\mathcal{R}$, which act as a source of dominance resistance points. Solutions inside $\mathcal{R}$ have different mappings to $\mathcal{Y}$ from one another (unlike the approach illustrated in Fig. 3), and are non-dominated by other members of the same bounded area. However, assuming correct construction, they will always be dominated by a solution in $\mathcal{P}$. Illustrations of some such problems are provided in Fig. 4, for $D=3$ and $D=4$, with a disconnected $\mathcal{P}$. The panels show situations where $\mathcal{R}$ is contiguous (in $\mathcal{X}$ ) to $\mathcal{P}$, and where they are disconnected from one another. Care needs to be taken when constructing the dominance resistance ar-

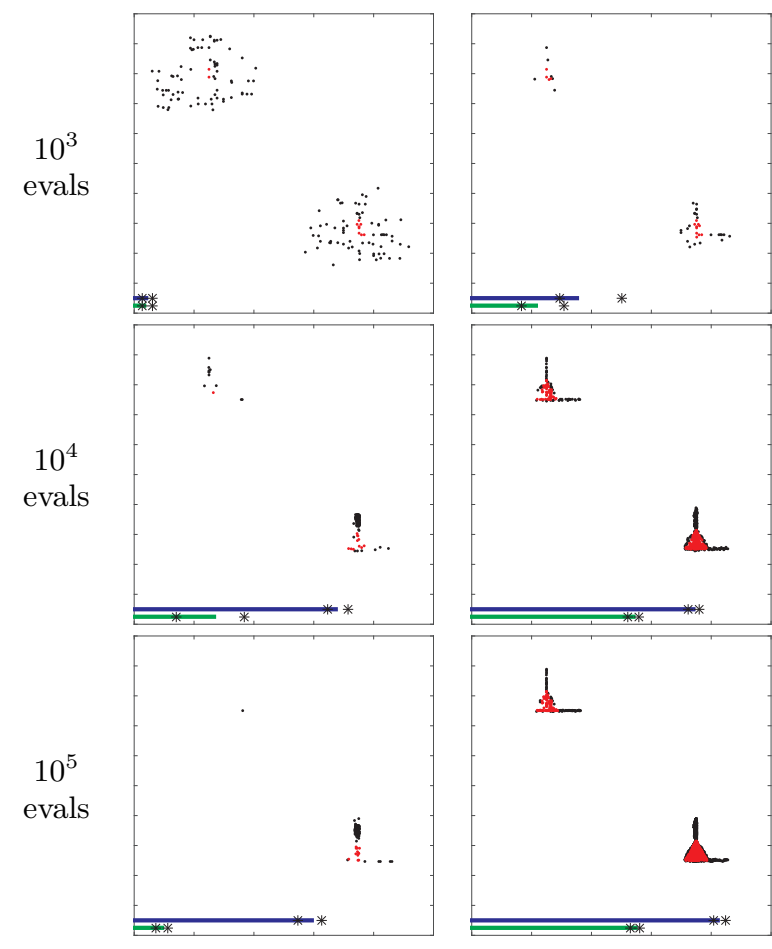

Figure 7: NSGA-II, example results on problem 1. Search population (left) and passive non-dominated archive member locations (right) in $\mathcal{X}$ after 1,000 , 10,000 and 100,000 function evaluations (top to bottom). Horizontal bars at the bottom indicate the proportion of $\mathcal{P}$ solutions in the population of the median run (lower bar), and on the reduced problem that omits the dominance resistance vertices (upper bar). Stars either side of the righthand side bar ends indicate inter-quartile ranges over 30 runs.

eas - the easiest way to ensure that a dominance resistance area is not accidentally constructed such that it hold Pareto optimal solutions, is to have the same relative positioning of vertices as in the Pareto optimal regions (bar the one or more omitted vertices).

Fig. 5 shows an eight-objective problem. Here there are seven dominance resistance areas, each involving fewer and fewer objectives. The cost landscape induced by each $\left|V_{d}\right|$ is shown in the panels in Fig. 6. The locations of the vertices in the test problems shown in Figs. 4-5 are detailed in Table 1 , and are used in the following empirical section.

\section{EMPIRICAL EXAMPLES}

We now provide examples of the performance of two popular MOEAs, which are widely used by the community, on distance-based problems constructed using the methodology described above. These are the Non-dominated Sorting Genetic Algorithm-II (NSGA-II) [1] and the Indicator Based Evolutionary Algorithm - with the $\epsilon+$ indicator $\left(\mathrm{IBEA}_{\epsilon+}\right)[22]$. We use three, four and eight objective variants in $K=2$. For the $D=3$ problem the search population size is set at 150 , for the $D=4$ problem 250 and for the $D=8$ problem 500. Other parameters are as recommended in the original papers. A passive non-dominated archive was 


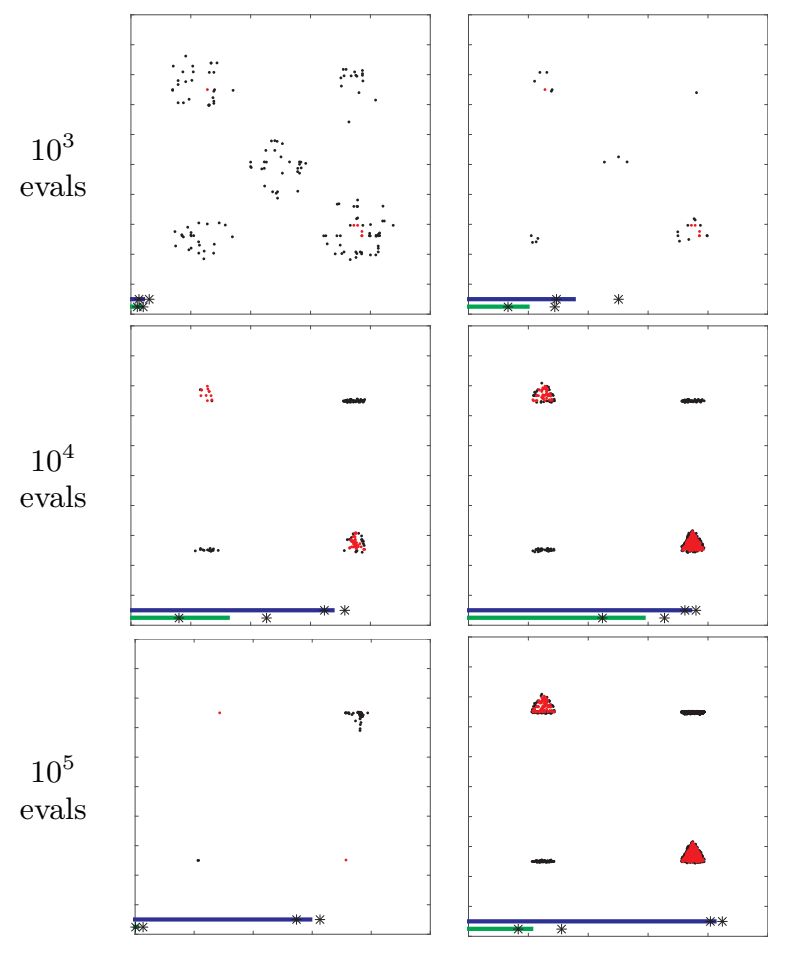

Figure 8: NSGA-II, example results on problem 2.
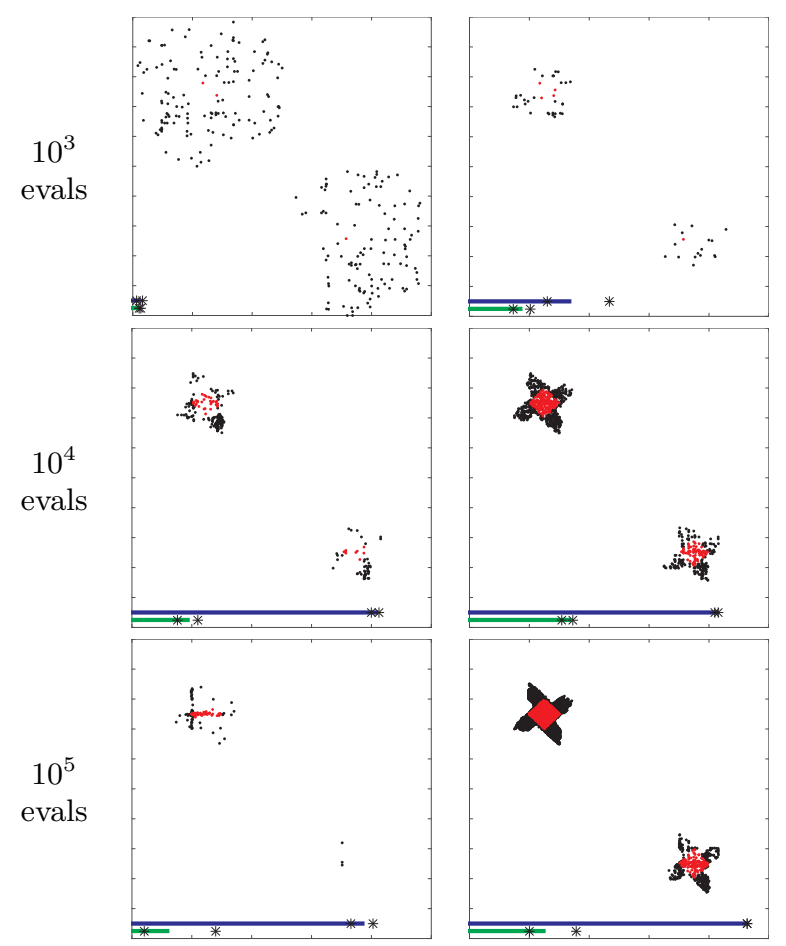

Figure 9: NSGA-II, example results on problem 3.

preserved for each algorithm, as recommended in [19]. This archive was unconstrained, thus accurately representing the non-dominated solutions found by an algorithm during dur-

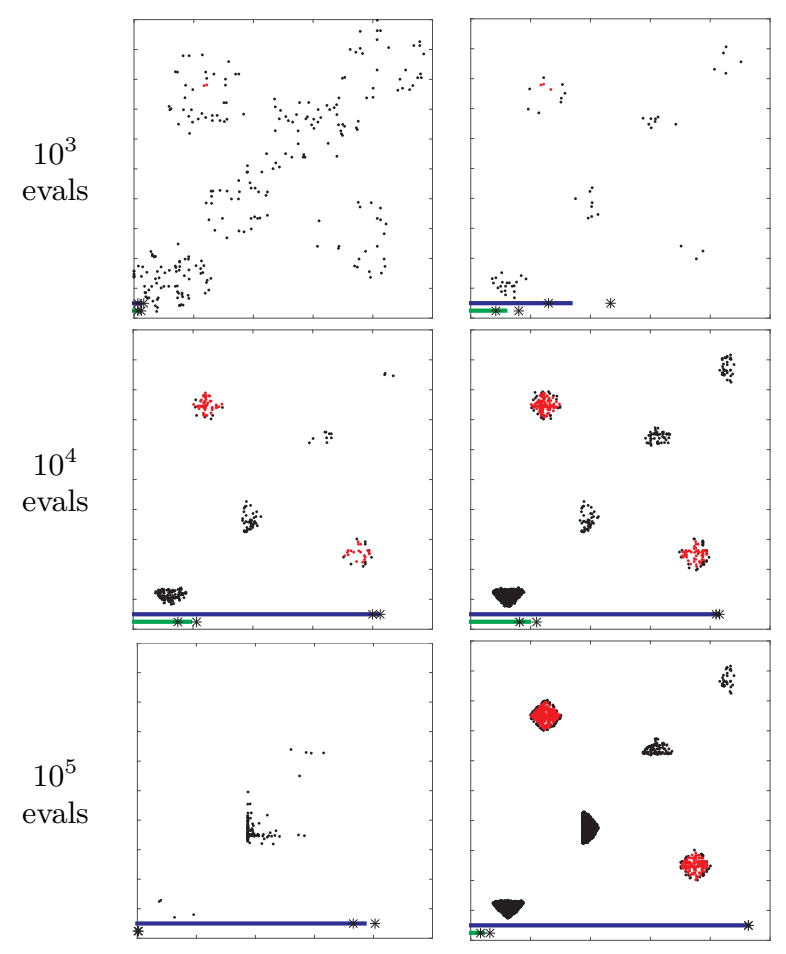

Figure 10: NSGA-II, example results on problem 4.

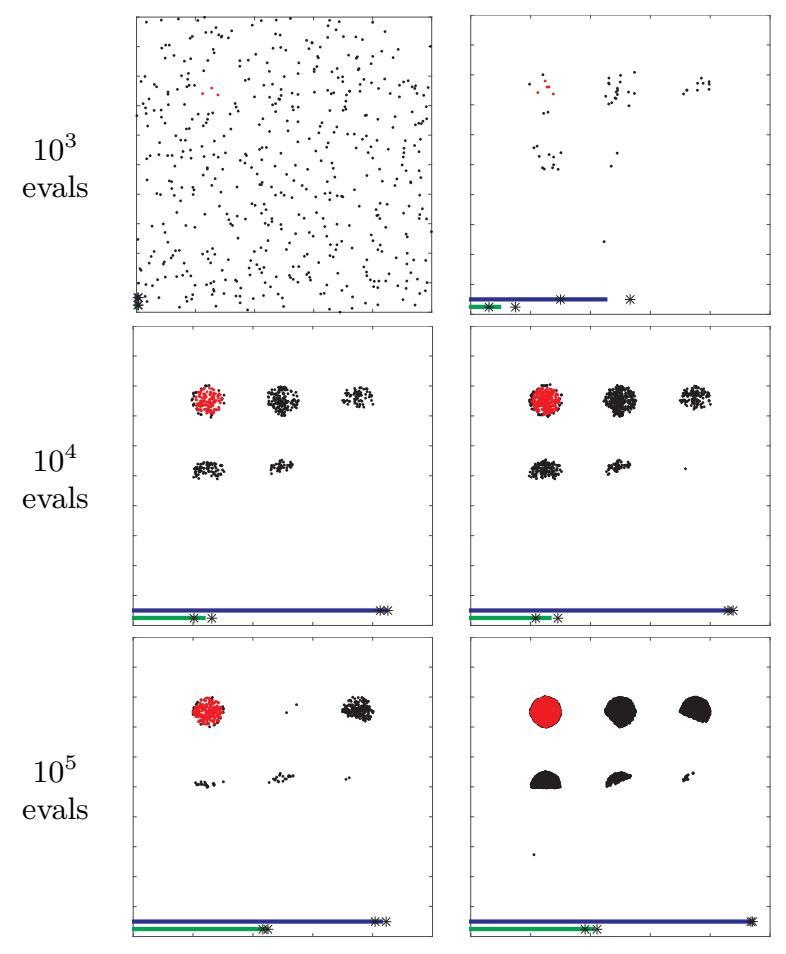

Figure 11: NSGA-II, example results on problem 5.

ing its search [3]. As well the membership of the search population, membership of this passive archive is also plotted in $\mathcal{X}$. This is important, as visualising the search population 


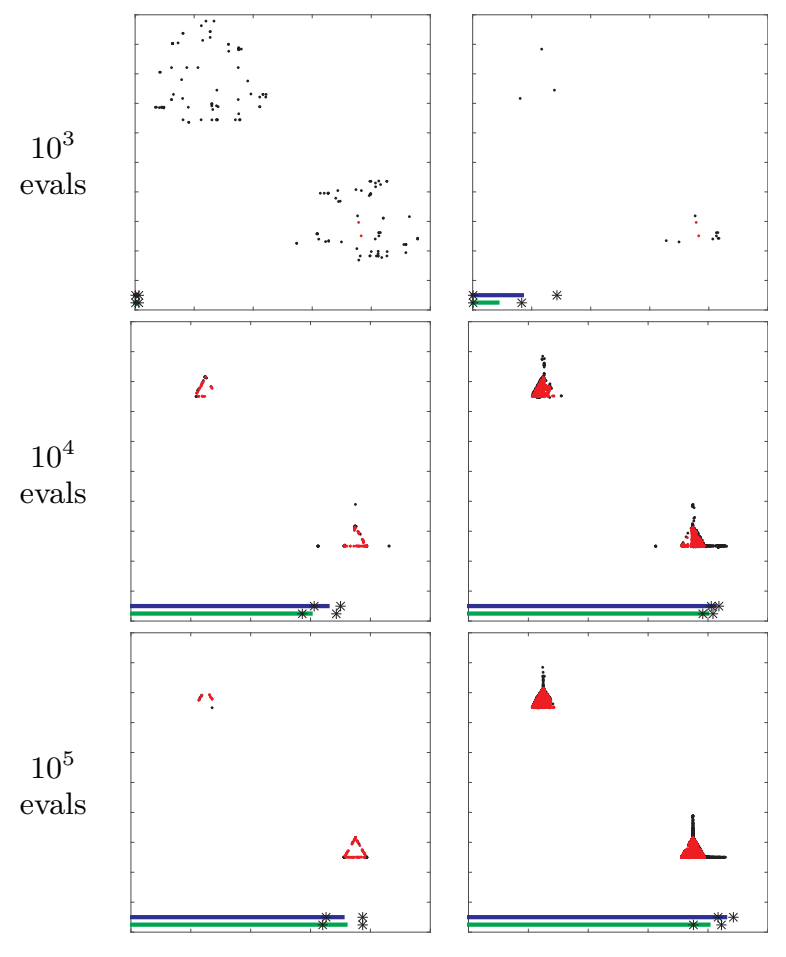

Figure 12: $\operatorname{IBEA}_{\epsilon+}$, example results on problem 1.

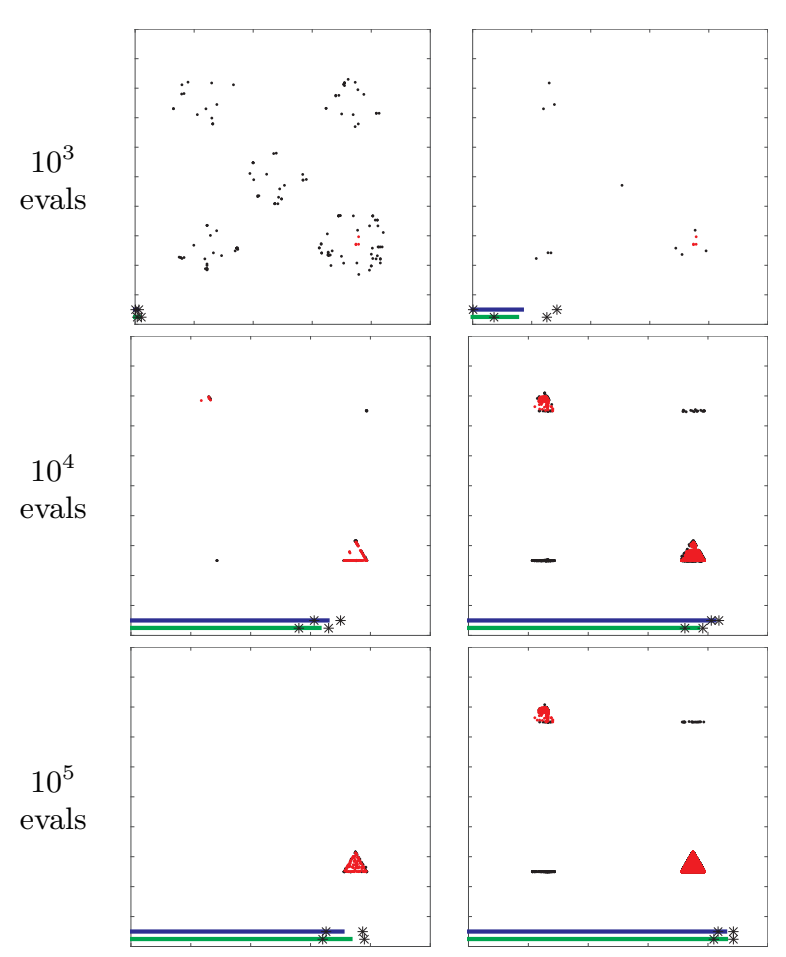

Figure 13: IBEA $_{\epsilon+}$, example results on problem 2.

at any particular time step simply provides a snapshot of the search process, whereas an unconstrained passive archive

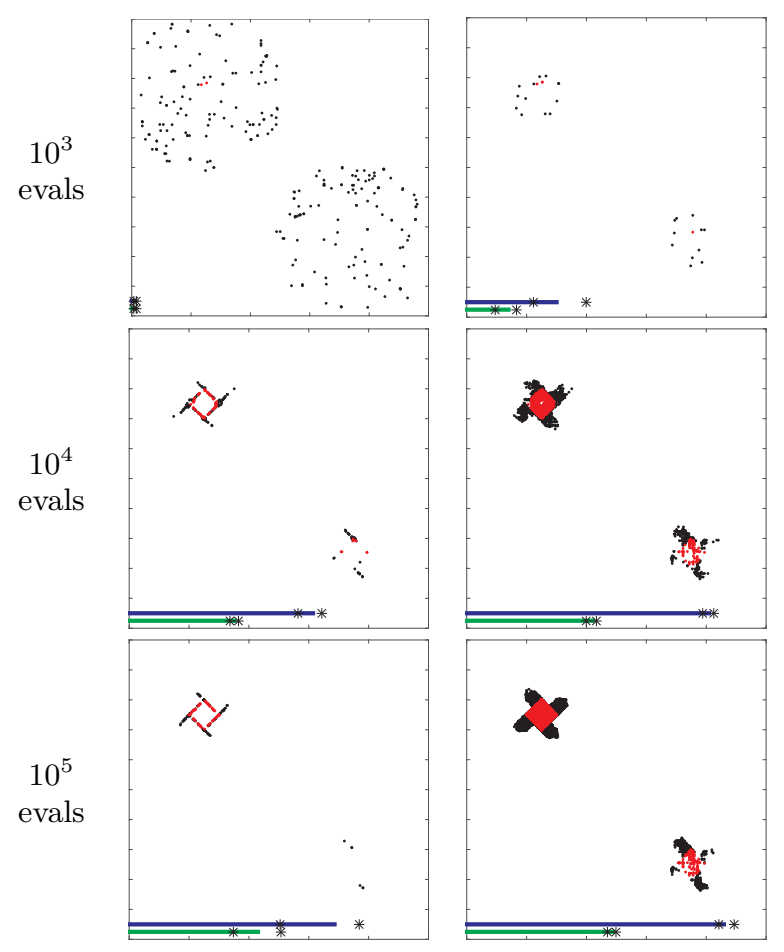

Figure 14: $\operatorname{IBEA}_{\epsilon+}$, example results on problem 3.

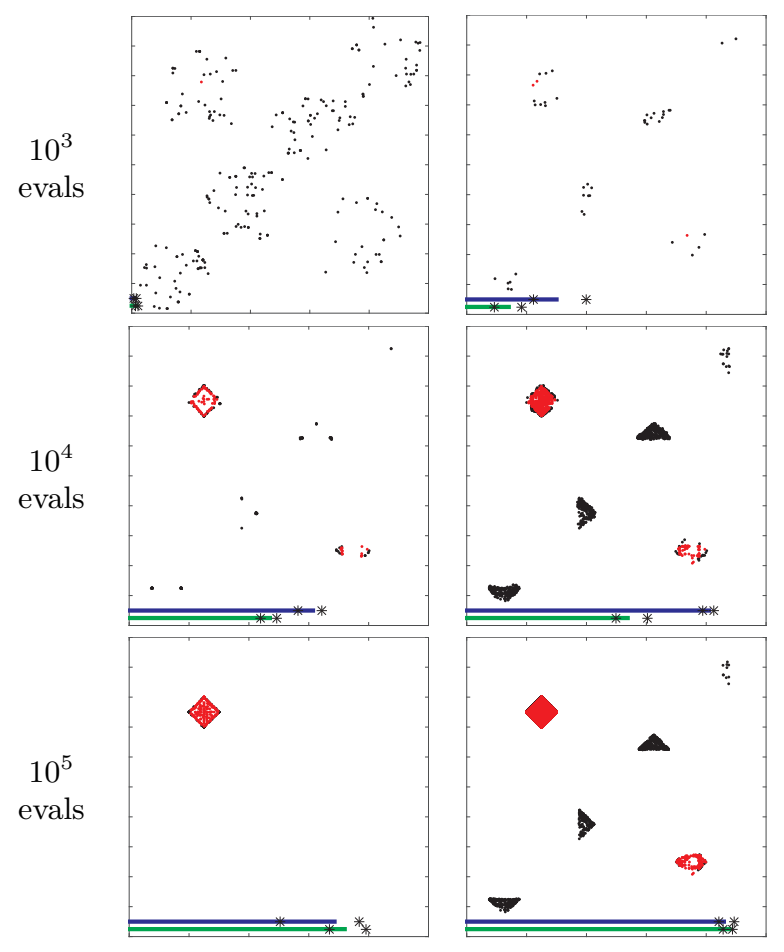

Figure 15: IBEA $_{\epsilon+}$, example results on problem 4.

shows all the best solutions an algorithm has discovered up until that point - giving insight into the search history. ${ }^{1}$

${ }^{1}$ MATLAB implementations and scripts to recreate the results may be found at https://github.com/fieldsend. 


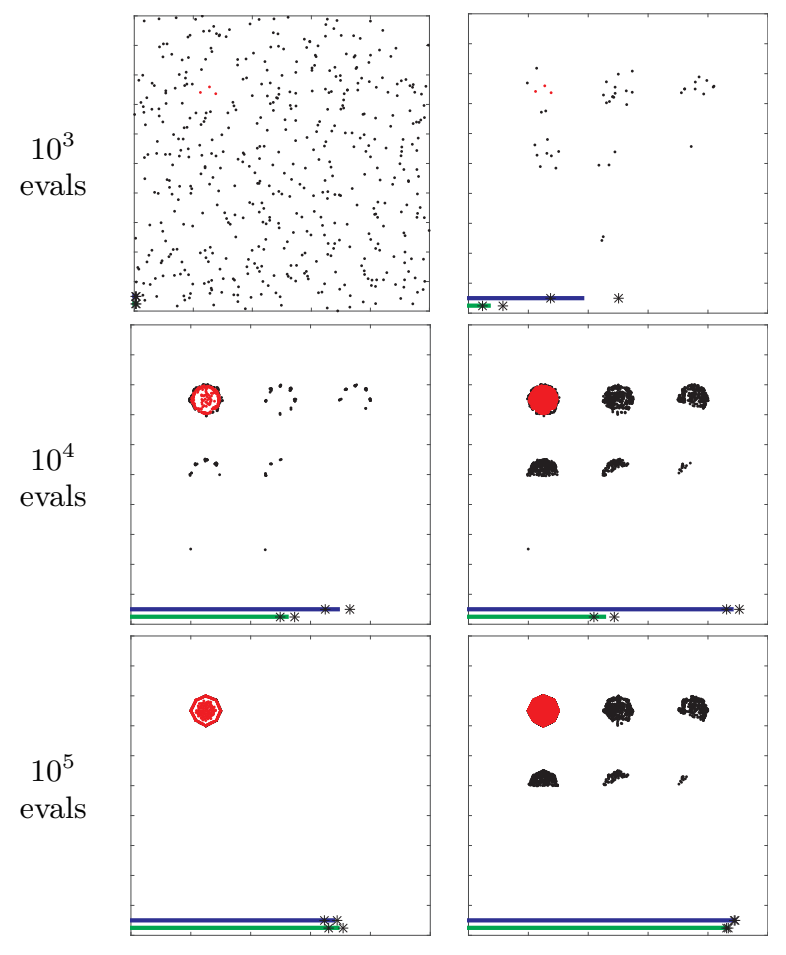

Figure 16: $\operatorname{IBEA}_{\epsilon+}$, example results on problem 5.

Results are provided in a consistent way for all problems, and algorithms. Each sub-panel in the results plots (Figs. 7-16) shows the location of an indicative search population (left panels) and a corresponding passive unconstrained nondominated archive (right panels). Solutions are plotted as red dots in $\mathcal{X}$ if they reside in $\mathcal{P}$, otherwise they are plotted as black dots. Results are shown for the algorithms after $10^{3}, 10^{4}$ and $10^{5}$ function evaluations. ${ }^{2}$ At the bottom of each panel are two horizontal bars starting at the y-axis. The lower (green) bar shows the median proportion of solutions in the corresponding population (over 30 runs) which reside in $\mathcal{P}$ at the plotted number of function evaluations. A bar spanning $70 \%$ of the panel width would therefore denote $70 \%$ of the solutions in the corresponding population of the median run (search population or passive archive, depending on panel) lie in $\mathcal{P}$. The upper (blue) bar shows the same information for 30 runs on identical problems which omit the vertices designed to cause dominance resistance points (i.e, for these reduced problems 1-4 $\left|V_{d}\right|=2$, and for problem 5 $\left.\left|V_{d}\right|=1\right)$. This enables us to quantify the effect of the dominance resistance elements of the problems on an algorithm's ability to find (and retain) solutions in $\mathcal{P}$. The stars plotted straddling the righthand side of the bar indicate the inter quartile ranges from the 30 runs.

Figs. 7-11 show the results for a typical run of NSGA-II on the five problems. By examining the location of the search population (left panels) and a passive unconstrained nondominated archive (right panels) it is interesting to see that the dominance resistant areas in particular cause NSGA-II problems. The search population hones in on the Pareto

\footnotetext{
${ }^{2}$ Or the closest number after this value, that is divisible by
} the search population size. regions by $10^{4}$ evaluations on problems 2 and 4 , but by $10^{5}$ evaluations the search population is focused almost exclusively on the suboptimal dominance resistance points. Even for problems 1 and 3 the search population has a tendency to been drawn to the contiguous $\mathcal{R}$ and has shrunk down to one of the two main regions. Compared to the problems without dominance resistance regions, we can see the proportion of $\mathcal{P}$ members in both populations tends to be much worse. We hypothesise that this behaviour is due to the niching approach used by NSGA-II. When the population is full of solutions with the best rank in this algorithm, the distance to the next nearest member in objective space is used for pruning. This removes the most tightly packed solutions in $\mathcal{Y}$, which tend to be solutions in $\mathcal{P}$ rather than $\mathcal{R}$ as they tend to be closer than solutions in $\mathcal{R}$. As more solutions are found in $\mathcal{R}$ (for which a corresponding dominating solution in $\mathcal{P}$ has not been found), the solutions in $\mathcal{P}$ are progressively 'crowded out' when using this niching approach.

Figs. 12-16 show the results for a typical run of IBEA $_{\epsilon+}$ on the five problems. IBEA $\mathrm{I}_{\epsilon}$ copes with the dominance resistance points better (on these particular problems). Here the $\mathcal{R}$ members are disadvantaged with respect to $\mathcal{P}$ members, as even if a corresponding dominating element of $\mathcal{P}$ has not been found, the $\epsilon+$ indicator penalises $\mathcal{R}$ members more as (at least) one of the objectives will always be much poorer than $\mathcal{P}$ elements in these problems (needing a larger $\epsilon$ to offset). By $10^{5}$ evaluations only the edges of $\mathcal{R}$ in problem 3 seem to be still be drawing the algorithm away from $\mathcal{P}$. In general the search population tends not to be overly swayed toward the dominance resistance areas. However, note that many elements from $\mathcal{R}$ do appear in the passive archive, meaning they have not been dominated by the found elements in $\mathcal{P}$ - the search process of $\mathrm{IBEA}_{\epsilon+}$ tends not to be pulled toward them once solutions found in $\mathcal{P}$ grow. On the other hand, the algorithm does seem to have a problem maintaining distinct disconnected regions of $\mathcal{P}$, often 'losing' one of the disconnected regions in problems 1-4 from the search population. This results in one optimal region in $\mathcal{X}$ being well-filled out in the passive archive, but the other often sparsely populated.

\section{SUMMARY}

We show how dominance resistance may be incorporated into the distance-based problem framework for generating test problems whose decision vectors are two-dimensional, making the search space easy to visualise. Previously sets of vertices per objective in these problems have been used to generate spatially distinct regions of design space which map to the same optimal locations in objective space (or in the case of [6], to represent a map-based distance problem). We have shown that sets of vertices may also be utilised to generate a source of dominance resistance points. We have illustrated how the search population of two popular evolutionary optimisers are affected by this modification, and on the best solutions they encounter, via the visualisation of both the search population and an unconstrained passive archive. The niching method in NSGA-II seems especially fragile to dominance resistance points generated by our test problems. It would be interesting to analyse the effects on different niching approaches further. Depending on the propensity of dominance resistant points in the real world problem at hand, this effect would appear to be quite 
deleterious to the search process. IBEA $\mathrm{I}_{\epsilon}$ appears less affected by the form of dominance resistance that the five test problems posed, although it tended to have a problem retaining spatially separated locations of the Pareto set.

We illustrated the problems in two-dimensional design space, however the framework described in [15] can be used to map these problems to arbitrarily large design spaces. We also note that there are conceptual similarities between dominance resistance areas, and the properties required to generate deceptive fronts in a search landscape. Therefore a similar route may also be use to embedding this property into easily visualisable distance-based test problems.

\section{ACKNOWLEDGMENTS}

This work was supported by the Engineering and Physical Sciences Research Council [grant number EP/M017915/1].

\section{REFERENCES}

[1] K. Deb, S. Agrawal, A. Pratap, and T. Meyarivan. A Fast Elitist Non-Dominated Sorting Genetic Algorithm for Multi-Objective Optimization: NSGA-II. In Parallel Problem Solving from Nature, pages 849-858. Springer, 2000.

[2] J. E. Fieldsend and R. M. Everson. Visualising high-dimensional Pareto relationships in two-dimensional scatterplots. In Evolutionary Multi-criterion Optimization, EMO 2013, pages 558-572, 2013.

[3] J. E. Fieldsend, R. M. Everson, and S. Singh. Using Unconstrained Elite Archives for Multi-Objective Optimisation. IEEE Transactions on Evolutionary Computation, 7(3):305-323, 2003.

[4] T. Hanne. On the convergence of multi objective evolutionary algorithms. European Journal of Operational Research, 117:553-564, 1999.

[5] F. W. Hewes and H. Gannett. Scribner's statistical atlas of the United States, showing by graphic methods their present condition and their political, social and industrial development. New York, C. Scribner's sons, 1883.

[6] H. Ishibuchi, N. Akedo, and Y. Nojima. A Many-Objective Test Problem for Visually Examining Diversity Maintenance Behavior in a Decision Space. In Genetic and Evolutionary Computation Conference, GECCO'11, pages 649-656, 2011.

[7] H. Ishibuchi, N. Akedo, H. Ohyanagi, Y. Hitotsuyanagi, and Y. Nojima. Many-Objective Test Problems with Multiple Pareto Optimal Regions in a Decision Space. In IEEE Symposium on Computational Intelligence in Multicriteria Decision-Making (MDCM), pages 113-120, 2011.

[8] H. Ishibuchi, N. Akedo, H. Ohyanagi, and Y. Nojima. Behavior of EMO Algorithms on Many-Objective Optimization Problems with Correlated Objectives. In IEEE Congress on Evolutionary Computation (CEC), pages 1465-1472, 2011.

[9] H. Ishibuchi, M. Yamane, N. Akedo, and Y. Nojima. Many-Objective and Many-Variable Test Problems for Visual Examination of Multiobjective Search. In IEEE Congress on Evolutionary Computation (CEC), pages 1491-1498, 2013.
[10] M. Köppen, R. Vicente-Garcia, and B. Nickolay. Fuzzy-Pareto-Dominance and its Application in Evolutionary Multi-Objective Optimization. In Evolutionary Multi-Criterion Optimization, pages 399-412. Springer Berlin Heidelberg, 2005.

[11] M. Köppen and K. Yoshida. Substitute Distance Assignments in NSGA-II for handling Many-objective Optimization Problems. In Evolutionary Multi-Criterion Optimization (EMO), number 4403 in Lecture Notes in Computer Science, pages 727-741, 2007.

[12] M. Köppen and K. Yoshida. Visualization of Pareto-sets in evolutionary multi-objective optimization. In Proceedings of the 7th International Conference on Hybrid Intelligent Systems, pages 156-161, 2007.

[13] M. Li, S. Yang, and X. Liu. A Test Problem for Visual Investigation of High-Dimensional Multi-Objective Search. In IEEE Congress on Evolutionary Computation (CEC), pages 2140-2147, 2014.

[14] M. Li, S. Yang, X. Liu, and R. Shen. A Comparative Study on Evolutionary Algorithms for Many-Objective Optimization. In Evolutionary Multi-Criterion Optimization, EMO 2013, number 7811 in Lecture Notes in Computer Science, pages 216-275, 2013.

[15] H. Masuda, Y. Nojima, and H. Ishibuchi. Visual Examination of the Behavior of EMO Algorithms for Many-Objective Optimization with Many Decision Variables. In IEEE Congress on Evolutionary Computation (CEC), pages 2633-2640, 2014.

[16] A. Pryke, S. Mostaghim, and A. Nazemi. Heatmap visualization of population based multi objective algorithms. In Evolutionary Multi-Criterion Optimization, number 4403 in Lecture Notes in Computer Science, pages 361-378, 2007.

[17] H. K. Singh, A. Isaacs, T. Ray, and W. Smith. A study on the performance of substitute distance based approaches for evolutionary many objective optimization. In Simulated Evolution and Learning, pages 401-410. Springer Berlin Heidelberg, 2008.

[18] P. H. A. Sneath. The application of computers to taxonomy. Journal of General Microbiology, 17(1):201-226, 1957.

[19] D. van Veldhuizen and G. Lamont. Multiobjective Evolutionary Algorithms: Analyzing the State-of-the-Art. Evolutionary Computation, 8(2):125-147, 2000.

[20] D. J. Walker, R. M. Everson, and J. E. Fieldsend. Visualizing mutually nondominating solution sets in many-objective optimization. IEEE Transactions on Evolutionary Computation, 17(2):165-184, 2013.

[21] J. Xu, K. Deb, and A. Gaur. Identifying the Pareto-Optimal Solutions for Multi-point Distance Minimization Problem in Manhattan Space. Technical Report COIN Report Number 2015018, Michigan State University, 2015.

[22] E. Zitzler and S. Künzli. Indicator-Based Selection in Multiobjective Search. In Parallel Problem Solving from Nature, pages 832-842. Springer, 2004. 